\title{
FACTORS OF TOEPLITZ FLOWS AND OTHER ALMOST 1-1 EXTENSIONS OVER GROUP ROTATIONS
}

\author{
T. DOWNAROWICZ and F. DURAND*
}

\begin{abstract}
If a minimal topological flow admits a symbolic extension then it also admits a symbolic almost 1-1 extension. The factors of symbolic almost automorphic flows are characterized as those almost automorphic flows which admit a symbolic extension. As an application, we provide concrete examples of factors of Toeplitz flows, which are neither Toeplitz flows nor odometers.
\end{abstract}

\section{Introduction}

From the topological point of view Toeplitz flows are characterized by the following three properties:

(a) being minimal,

(b) being almost 1-1 extensions of odometers,

(c) being symbolic.

The first and last property have been known since the time when Jacobs and Keane started to investigate these flows ([11]). The property (b) was described by Eberlein ([7]), one year later. Sufficiency of these three conditions has been established in [12] (see [6] for another proof).

In this paper we provide a similar conjunction of three conditions which characterizes all topological factors of Toeplitz flows. Namely, the conditions (a) and (b) remain unchanged, while (c) has to be replaced by the condition of admitting any symbolic extension.

The above is attained by applying two much more general theorems. The first one concerns factors of arbitrary minimal almost 1-1 extensions over group rotations, and it says that every such flow is itself an almost 1-1 extension over a group rotation. This fact can be derived from the characterization of so called almost automorphic points presented in [8]. In order to make this paper self-contained, we provide a direct proof. The second theorem states that if a

\footnotetext{
* The research of the first author supported by the grant KBN 2 P03A 03915 [98-01 r.].

The authors would like to express their gratitude to the Institut de Mathématiques de Luminy, France, where the first draft of this paper was written.

Received January 12, 1999.
} 
minimal flow admits any symbolic extension then it also admits an almost 11 such extension. Similar theorems about replacing an arbitrary extension by an almost 1-1 extension have been known since a long time (e.g. [9]), but a general statement concerning particularly the symbolic extensions was missing. A symbolic version of the Furstenberg-Weiss theorem can be found in [6], alas, in that paper there are additional assumptions made on the base flow, and these assumptions are relatively strong from the point of view of our purposes (for instance being itself symbolic). Interestingly, the methods developed in this note allow to completely skip these assumptions in [6] and thus generalize the results stated there.

At the end we provide a practical method of producing flows satisfying our three conditions, and we give some examples exhibiting certain, perhaps unexpected, topological properties.

This study has been provoked by the question raised in [10] whether there exist totally disconnected factors of Toeplitz flows other than Toeplitz flows and odometers, for which we would like to express our gratitude to the authors of the above paper.

\section{Terminology}

In order to avoid confusion, we point out certain aspects of the terminology.

For example, like many authors, we will use the word "flow" with respect to what others call "cascades", i.e., to the action of the group Z of the integers. More specifically, a flow is a pair $(X, T)$, where $X$ is a compact metric space and $T: X \rightarrow X$ denotes a homeomorphism. Perhaps this is not the most apt choice, but the name "Toeplitz flows" has been used in this context since a long time. The other meaning of "flow", i.e., the continuous action of $R$, will not appear in this note.

Another non-uniformly called object is an "odometer". The frequently used synonyms are "adding machine" or " $p$-adic integers". In any case one has in mind a compact monothetic infinite totally disconnected group $G$. It is customary to use either of the first two names to denote the flow $(G, R)$ on $G$, where $R$ is the rotation by a topological generator of $G$. The last name involves a parameter $p$, which in the general case denotes an arbitrary sequence of integers, i.e., $p=\left(p_{t}\right)_{t \in \mathrm{N}}$. Then $G$ is equal to the inverse limit of the cyclic groups $Z_{q_{t}}$, where $q_{t}=p_{1} p_{2} \ldots p_{t}$. If not clearly specified, the name $p$-adic integers may suggest that the sequence $p_{t}$ is constant (or even equal to a prime number $p$ ) in which context it appears most often. In this paper we will stick to the first option (as it is the shortest) and indeed, we will always consider $G$ along with its natural action by $R$. All groups appearing in this paper will be denoted additively. 
We will often refer to "symbolic flows", by which we shall understand what others call "subshifts", i.e., shift invariant closed subsets of $\Sigma^{\mathrm{Z}}$ (the set $\Sigma$ called "alphabet" is necessarily finite) along with the action induced by the left shift, always denoted by $S$. It is well known that such flows are characterized in purely topological categories as expansive actions on totally disconnected spaces. The shift transformation $S$ has well defined meaning also in the case where $\Sigma$ is infinite, but then we no longer deal with a symbolic flow, and we will avoid using the letter $\Sigma$ in this context. When talking about a symbolic flow $(X, S)$, we will be using such phrases as "the block $b$ of length $k$ appears in $x \in X$ (starting) at the position (coordinate) $n$ ". This specifies that $x[n, n+k)=b$, where $b=b[0, k) \in \Sigma^{k}$.

For the purposes of this paper we will need neither the formal definition of an odometer nor that of a Toeplitz flow. It suffices to know that the first one is a minimal equicontinuous flow, and that the second is characterized by the three conditions stated at the beginning of this article. For the original definition we refer the reader to any paper where Toeplitz flows appear in the title.

Let $(Z, T)$ and $(Y, U)$ be two flows. We say that $(Y, U)$ is an "extension" of $(Z, T)$, or equivalently that $(Z, T)$ is a "factor" of $(Y, U)$ if there exists a continuous surjection $\pi: Y \rightarrow Z$ such that $\pi U=T \pi$. The last condition is usually pronounced as $\pi$ "preserves the action". If an extension is given, then by "fibers" we will mean preimages (by $\pi$ ) of single points.

An extension is called "almost 1-1" if the set of points having one-point preimages is residual (contains a dense $G_{\delta}$ ) in $Z$. In the minimal case it suffices to verify that a one-point fiber exists.

The flows which are minimal almost 1-1 extension of group rotations have been studied under the name "almost automorphic flows" ([12], [13], [8]). We will also use this name from time to time.

\section{Factors of almost automorphic flows}

LeMma 3.1. Let $G$ be a compact abelian group and let $K$ be a closed subset of $G$. If $g+K \subset K$ for some $g \in G$ then $g+K=K$.

Proof. First observe that the sequence $(n g)_{n \in \mathrm{N}}$ has a subsequence converging to the unity 0 of the group $G$ (take $\left(n_{k+1}-n_{k}\right) g$, where $n_{k} g$ is any convergent subsequence). Now, if $n_{k} g \rightarrow 0$, then $n_{k} g+K \subset g+K$ for each $k$ and these sets tend to $0+K=K$, hence $K \subset g+K$.

Theorem 3.2 ([8], Theorem 9.13 and Proposition 9.9). Let $(G, R)$ be a minimal rotation of a compact monothetic group, and let $(X, T)$ be a minimal almost $1-1$ extension of $(G, R)$ via a map $\pi$. Further, let $(Y, S)$ be an arbitrary factor of $(X, T)$ via a map $\phi$. Then there exists a factor $(H, \tilde{R})$ of $(G, R)$ (via 
some map $\psi)$ such that $(Y, S)$ is an almost $1-1$ extension of $(H, \tilde{R})$ (via some map $\rho)$. The corresponding diagram commutes:

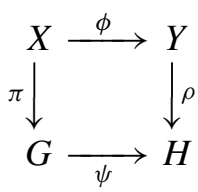

Proof. Recall that the Ellis semigroup $E(X, T)$ of the flow $(X, T)$ consists of all (not necessarily continuous) maps obtained as pointwise limits of the iterates of $T$. Minimality is equivalent to the condition that for any pair of points $x, x^{\prime} \in X$ there exists a $\tau \in E(X, T)$ with $\tau(x)=x^{\prime}$. For our monothetic group rotation, $E(G, R)$ consists of all rotations by the elements of $G$. If $\phi:(X, T) \rightarrow(Y, S)$ is a factor map, then for every $\sigma \in E(Y, S)$ there exists a $\tau \in E(X, T)$ such that $\phi \tau=\sigma \phi$. We then say that $\tau$ is a lift of $\sigma$. Conversely, every $\tau \in E(X, T)$ is a lift of a $\sigma \in E(Y, S)$. Such $\sigma$ is unique for $\tau$ and we call it the projection of $\tau$. It is straightforward that if $\tau$ is a lift of $\sigma$ and $y \in Y$ then $\tau \phi^{-1}(y) \subset \phi^{-1} \sigma(y)$.

For each $y \in Y$ we denote

$$
H_{y}=\pi \phi^{-1}(y) .
$$

Clearly $\left(H_{y}\right)_{y \in Y}$ is a cover of $G$ by closed sets. We claim that for any two such sets $H_{y}, H_{y^{\prime}}$ there exists a $g \in G$ such that $H_{y}=g+H_{y^{\prime}}$. Indeed, let $\sigma \in E(Y, S)$ be such that $\sigma(y)=y^{\prime}$, let $\tau$ be a lift of $\sigma$ and let the rotation by $g_{1} \in G$ be the projection of $\tau$. Then

$$
H_{y^{\prime}}=\pi \phi^{-1} \sigma(y) \supset \pi \tau \phi^{-1}(y)=g_{1}+\pi \phi^{-1}(y)=g_{1}+H_{y} .
$$

By a symmetric argument, $H_{y} \supset g_{2}+H_{y^{\prime}}$ for some $g_{2} \in G$. Finally,

$$
H_{y} \supset g_{2}+H_{y^{\prime}} \supset g_{2}+g_{1}+H_{y} \text {. }
$$

By Lemma 3.1, we have equalities, hence we can assign $g=g_{2}$.

Next we show that two such sets $H_{y}, H_{y^{\prime}}$ are either disjoint or equal. Suppose that there is a $g \in H_{y} \cap H_{y^{\prime}}$. Fix a point $e \in G$ with a one-point fiber $\pi^{-1}(e)$, Let $\tau \in E(X, T)$ be a lift of the rotation by $e-g$ and let $\sigma \in E(Y, S)$ be the projection of $\tau$. We have

$$
e \in e-g+H_{y}=\pi \tau \phi^{-1}(y) \subset \pi \phi^{-1} \sigma(y),
$$

and analogously for $y^{\prime}$. Thus $\pi^{-1}(e)$ intersects both $\phi^{-1} \sigma(y)$ and $\phi^{-1} \sigma\left(y^{\prime}\right)$. But since it is a one-point fiber, the last two sets (being non-disjoint preimages 
of points) must be equal. Passing to the image by $\pi$ we obtain

$$
H_{\sigma(y)}=H_{\sigma\left(y^{\prime}\right)} .
$$

We have proved that

$$
e-g+H_{y} \subset H_{\sigma(y)}=H_{\sigma\left(y^{\prime}\right)} \supset e-g+H_{y^{\prime}} .
$$

Since these are all rotations of the same set, we have equalities.

It now follows that the sets $H_{y}$ form a quotient group $H$ of $G$. (The $H_{y}$ which contains the unity of $G$ is easily seen to be a closed subgroup.) Thus $(H, \tilde{R})$ (where $\tilde{R}$ denotes the rotation induced on $H$ by $R$ ) is a topological factor of $(G, R)$. We skip the standard argument showing that the map $\rho: Y \rightarrow H$ defined by $\rho(y)=H_{y}$ is continuous and that the corresponding diagram commutes. The last thing we need is that $\rho$ provides an almost $1-1$ extension. To this end, by minimality, it suffices to find a one-point fiber of $\phi$. Consider a point $y$ for which $e \in H_{y}=\rho(y)$. Then $\pi^{-1}(e) \in \phi^{-1}(y)$, which determines $y$ as a unique such point.

\section{Symbolic almost 1-1 extensions}

Denote by $\mathscr{A}$ the class of all almost automorphic flows. We are especially interested in characterizing the class $\mathscr{F} \mathscr{S} \mathscr{A}$ of all flows which can be obtained as factors of symbolic flows from $\mathscr{A}$. From Theorem 3.2 it follows that (including finite flows) every such flow is again in $\mathscr{A}$. Let $\mathscr{F} \mathscr{S}$ denote the class of all minimal flows which admit a symbolic extension, and $\mathscr{F} \mathscr{S}^{\prime}$, those which admit a minimal almost 1-1 symbolic extension. Since a composition of almost 1-1 extensions is an almost 1-1 extension, we have $\mathscr{A} \cap \mathscr{F} \mathscr{S}^{\prime} \subset \mathscr{F} \mathscr{S} \mathscr{A} \subset \mathscr{A} \cap \mathscr{F} \mathscr{S}$. There are no immediate reasons why we could reverse any one of the above inclusions. The following theorem solves the problem, by showing that $\mathscr{F} \mathscr{S}=\mathscr{F} \mathscr{S}^{\prime}$.

Note that if a minimal flow admits an extension with certain properties (such as being almost $1-1$ or symbolic), then it also admits a minimal extension with the same properties (namely a minimal subset of the extension), thus in the assertion of our theorem we can skip minimality of the extensions of minimal flows.

THEOREM 4.1. Let $(Z, T)$ be a minimal flow and $(X, S)$ a symbolic extension of $(Z, T)$. Then there exists a symbolic almost $1-1$ extension $(Y, S)$ of $(Z, T)$.

Proof. We employ a modified version of the method used in [6] where an almost $1-1$ extension of a minimal flow $(Z, T)$ is constructed from an arbitrary extension with some additional conditions on $(Z, T)$ (one possible condition 
was that $(Z, T)$ is symbolic). Applying an idea, due to Y. Lacroix, of marking the return times of trajectories to certain open sets we introduce a symbolic "almost factor" $(\tilde{Z}, S)$ of $(Z, T)$ through which we are now able to generalize the technique of [6]. The main difference is that, unlike in [6], there is no immediate way of defining the factor map from $Y$ to $Z$. Most of the difficulty of the proof arises from the necessity of such assignment.

On the other hand, we have simplified slightly the construction by skipping the technical details responsible for minimality of the flow $(Y, S)$. We do not need to care about minimality, because, as mentioned before, any minimal subset of $(Y, S)$ is again an almost $1-1$ extension of $(Z, T)$.

Denote by $\pi$ the factor map from $X$ to $Z$. Let $\left(U_{t}\right)_{t \in \mathbb{N}}$ be a decreasing sequence of open sets with a one-point intersection $z_{0} \in Z$. Since the theorem holds trivially in finite spaces, we are assuming that there are no isolated points in $Z$, and that the sets $U_{t}$ essentially decrease. For each $z \in Z$ we produce a $0-1$-sequence $\tilde{z}$ which will mark the return times of the trajectory of $z$ to the sets $U_{t}$. This sequence will be constructed inductively.

Initially $\tilde{z}$ consists entirely of zeros. Next we place the symbol 1 at the positions corresponding to the integer moments when the trajectory of $z$ visits $U_{1}$. By choosing $U_{1}$ small enough, we can assume that consecutive 1's are separated by at least two zeros. The blocks of the form $1000 \ldots 0$ we call $U_{1^{-}}$ blocks of type 0 . Later we will also use similar blocks of the form $1111 \ldots 10$ which we will call $U_{1}$-blocks of type 1 . Note that in any concatenation of $U_{1^{-}}$ blocks (of both types) we can determine the breaking points by locating the pairs 01 . Every $U_{1}$-block of type 0 can be promoted to a block of type 1 by replacing all but the last of its zeros by ones. At this stage $\tilde{z}$ consists entirely (as a concatenation) of $U_{1}$-blocks of type 0 , for example:

$\tilde{z}=\ldots \overbrace{1000}^{0} \overbrace{100000}^{0} \overbrace{100}^{0} \overbrace{10000}^{0} \overbrace{100}^{0} \overbrace{\overbrace{1000}^{0}}^{0} \overbrace{100000}^{0} \overbrace{1000}^{0} \overbrace{10000}^{0} \overbrace{1000}^{0} \overbrace{1000}^{0} \overbrace{100000}^{0} \ldots$

Next, we observe the integer moments when the trajectory of $z$ visits $U_{2}$. Since $U_{2} \subset U_{1}$, these integers meet some of the previously inserted in $\tilde{z}$ symbols 1 , i.e., the first positions in certain $U_{1}$-blocks of type 0 . We now promote these $U_{1}$-blocks to $U_{1}$-blocks of type 1 . After this step $\tilde{z}$ is a concatenation of $U_{1}$-blocks of both types, as in the example below:

$\tilde{z}=\ldots \overbrace{1110}^{1} \overbrace{100000}^{0} \overbrace{100}^{0} \overbrace{10000}^{0} \overbrace{110}^{1} \overbrace{1000}^{0} \overbrace{\overbrace{100000}^{0}}^{0} \overbrace{1110}^{1} \overbrace{10000}^{0} \overbrace{1000}^{0} \overbrace{1000}^{0} \overbrace{100000}^{0} \ldots$.

As noticed before, the above decomposition is uniquely determined.

Again, we can assume that consecutive $U_{1}$-blocks of type 1 are separated by at least two $U_{1}$-blocks of type 0 . We call $U_{2}$-blocks of type 0 the concatenations of $U_{1}$-blocks where the structure of types is $1000 \ldots 0$. Analogously, the structure of types of $U_{1}$-blocks in a $U_{2}$-block of type 1 is $1111 \ldots 10$. Each 
$U_{2}$-block of type 0 can be promoted to a $U_{2}$-block of type 1 by promoting all but last of its component $U_{1}$-blocks of type 0 . By the same argument as before, in any concatenation of $U_{2}$-blocks we can determine the breaking points by analyzing the sequence of types of the component $U_{1}$-blocks. At this stage $\tilde{z}$ consists entirely of $U_{2}$-blocks of type 0 . In our example this partition looks as follows:

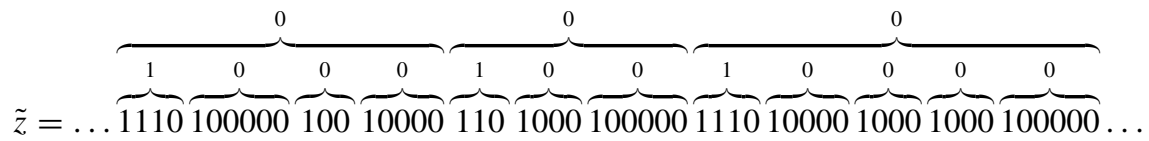

Next we mark the return times of $z$ to $U_{3}$ by promoting certain $U_{2}$-blocks in appropriate places, for example:

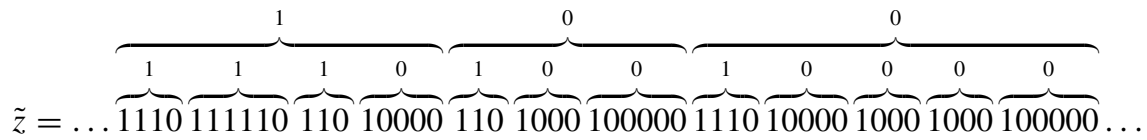

And so on. We omit the formal description of the obvious induction. In step $t+1$ we promote certain $U_{t}$-blocks of type 0 to mark the return times to $U_{t+1}$. Promoting a $U_{t}$-block consists in promoting all but the last of its component $U_{t-1}$-blocks of type 0 , (hence it is recursively defined).

In the limit (which clearly exists, because we never replace ones by zeros) we obtain a $0-1$-sequence $\tilde{z}$ having the following properties:

(.) for each $t, \tilde{z}$ is a concatenation of $U_{t}$-blocks,

(.) each $U_{t}$-block consists of at least three $U_{t-1}$-blocks, the first of type 1, the last of type 0 ,

(.) the partition of $\tilde{z}$ into $U_{t}$-blocks is unique,

(.) the starting positions of the component $U_{t}$-blocks coincide with the return times of $z$ to the set $U_{t}$.

If a $U_{t}$-block consists of $n U_{t-1}$-blocks, then the one whose index is $\frac{n}{2}$ or $\frac{n+1}{2}$ will be referred to as the central $U_{t-1}$-block. The central position of a $U_{t}$-block is defined recursively as the central position in its central component $U_{t-1}$-block.

The closure $\tilde{Z} \subset\{0,1\}^{Z}$ of the collection of all so obtained sequences $\tilde{z}$ is shift invariant, but it is not a factor of $(Z, T)$. The map $z \rightarrow \tilde{z}$ fails to be continuous at points whose trajectories visit the boundaries of the sets $U_{t}$. Neither is in general $(\tilde{Z}, S)$ an extension of $(Z, T)$. (In cases where it is, we can end the proof here, because then this extension is almost 1-1. Alas, this happens only in very restricted cases, e.g., if $(Z, T)$ is equicontinuous). Nevertheless, $\tilde{Z}$ will play an important role in our construction. 
From now on, by $U_{t}$-blocks (of both types), we shall understand only those $U_{t}$-blocks which do appear in $\tilde{Z}$.

We will treat the sets $U_{t}$ differently for even and odd indices $t$. Therefore we will change slightly our notation. Namely we assume that we have two sequences $U_{t}$ and $V_{t}\left(U_{t} \supset V_{t} \supset U_{t+1}, t \in \mathrm{N}\right)$. Accordingly, we will speak about $U_{t}$-blocks and $V_{t}$-blocks. We need to be more specific about the choice of the sets $U_{t}$ and $V_{t}$. Fix a summable sequence $\left(\epsilon_{t}\right)$. For each given $t$ we pick $U_{t}$ of diameter smaller than $\epsilon_{t}$. Then, by minimality, there exists a positive integer $p_{t}$ for which

(1) $U_{t}, T\left(U_{t}\right) \ldots, T^{p_{t}}\left(U_{t}\right)$ is a cover of $Z$,

and at the same time such that for each pair of points $x_{1}, x_{2} \in X$

(2) $x_{1}\left[-p_{t}, p_{t}\right]=x_{2}\left[-p_{t}, p_{t}\right] ; \Longrightarrow \operatorname{dist}\left(\pi\left(x_{1}\right), \pi\left(x_{2}\right)\right)<\epsilon_{t}$.

The choice of $V_{t}$ is even more sophisticated: Consider the family of sets consisting of $T^{-p_{t}}\left(U_{s}\right) \ldots, T^{p_{t}}\left(U_{s}\right)$ with $s \leq t$, and $T^{-p_{t}}\left(V_{s}\right) \ldots, T^{p_{t}}\left(V_{s}\right)$ with $s<t$, and let $\mathscr{V}_{t}$ be the finest partition of $U_{t}$ generated by the intersections of these sets and their complements. At least one of the elements of $\mathscr{V}_{t}$ has nonempty interior. We decide to choose $V_{t}$ so that

(3) $\bar{V}_{t}$ is contained in the interior of a set from $\mathscr{V}_{t}$. and

(4) $T^{-p_{t}}\left(V_{t}\right) \ldots, T^{p_{t}}\left(V_{t}\right)$ have diameters smaller than $\epsilon_{t}$, By choosing $V_{t}$ sufficiently small we can assume that

(5) the sets $V_{t}, T\left(V_{t}\right) \ldots, T^{\left(4 p_{t}+3\right)} p_{t}\left(V_{t}\right)$ are pairwise disjoint.

By (1), all $U_{t}$-blocks are not longer than $p_{t}$, by (5), all $V_{t}$-blocks are not shorter than $\left(4 p_{t}+3\right) p_{t}$, hence

(6) each $V_{t}$-block consists of at least $4 p_{t}+3 U_{t}$-blocks.

From the condition (3) it can be derived that

(7) all $V_{t}$-blocks have the same first component $U_{t}$-block, and all $V_{t}$-blocks have the same last component $U_{t}$-block.

Denote by $\Sigma$ the alphabet of $X$. As in [6], we treat each $x \in X$ as the top row, and we add two more rows below: the middle row temporarily entirely filled with empty cells (later in this row we will insert also letters from $\Sigma$ ), and the bottom row, where we place the sequence $\tilde{z}$, with $z=\pi(x)$. This produces a sequence $\tilde{x}$ over the finite alphabet $\tilde{\Sigma}=\Sigma \times(\Sigma \cup\{\square\}) \times\{0,1\}$ (the letters of this alphabet can be viewed as columns of height 3 ). The set of so obtained elements $\{\tilde{x}: x \in X\}$ is easily seen to be shift invariant. We denote by $\tilde{X}$ the closure of the above set in $\tilde{\Sigma}^{\mathrm{z}}$. Clearly, $(\tilde{X}, S)$ is a symbolic extension of 
$(X, T)$ (by the projection onto the top row), hence also of $(Z, T)$. We denote by $\tilde{\pi}$ the factor map from $(\tilde{X}, S)$ to $(Z, T)$.

Note that since we have taken the closure and since $z \rightarrow \tilde{z}$ is not continuous, it is no longer true that the bottom row of an arbitrary $\tilde{x} \in \tilde{X}$ coincides with $\tilde{z}$, where $z=\tilde{\pi}(\tilde{x})$. However, since the above does hold on a dense subset of $\tilde{X}$, it is true that for any $\tilde{x} \in \tilde{X}$ the bottom row is an element of $\tilde{Z}$, and, if $r$ is the starting position of a $V_{t}$-block in the bottom row of $\tilde{x}$, then

(8) $\tilde{\pi}(\tilde{x}) \in T^{-r}\left(\overline{V_{t}}\right)$,

while in the remaining cases

(9) $\tilde{\pi}(\tilde{x}) \notin T^{-r}\left(V_{t}\right)$.

By $t$-wagons we shall mean these blocks over the alphabet $\tilde{\Sigma}$ which have a $U_{t}$-block in its bottom row. Analogously, by $t$-trains we shall mean these blocks which have a $V_{t}$-block in its bottom row. Rereading (6), we obtain that

(6') each $t$-train is a concatenation of at least $4 p_{t}+3 t$-wagons.

We call the first and last wagons in each train the locomotive and the caboose, respectively. The condition (7) now means that

(7') all $t$-locomotives have identical bottom row, all $t$-cabooses have identical bottom row.

By (1),

(1') the lengths $l_{t}$ and $c_{t}$ of the $t$-locomotives and $t$-cabooses, respectively, are at most $p_{t}$.

By original $t$-trains we shall understand these $t$-trains which do appear in $\tilde{X}$.

Consider two points $\tilde{x}_{1}, \tilde{x}_{2} \in \tilde{X}$ having the same original $t$-train covering the zero coordinate. If the zero coordinate falls at least $p_{t}$ positions away from the ends of this $t$-train, then (2) applies to the top rows of $\tilde{x}_{1}$ and $\tilde{x}_{2}$. By the definition of $\tilde{\pi}$ we obtain that

(10) $\operatorname{dist}\left(\tilde{\pi}\left(\tilde{x}_{1}\right), \tilde{\pi}\left(\tilde{x}_{2}\right)\right)<\epsilon_{t}$.

In the remaining case we can apply (8) for some $r\left(-p_{t}<r<p_{t}\right)$, hence by (4) we conclude that (10) holds as well.

We will now inductively define a sequence of codes $\phi_{t}$ transforming the original $t$-trains into so called regular $t$-trains, preserving their lengths and the bottom row. We let $\phi_{0}$ be the identity.

Suppose that after step $t$ we have changed all $t$-trains of $\tilde{X}$ into regular $t$-trains by a 1-1 transformation $\phi_{t}$, which does not alter the bottom row. Moreover, we assume that the central cell in the middle row of every $t$-train remains empty. Clearly, the code $\phi_{t}$ can be applied in a natural way to any block or sequence which is a concatenation of original $t$-trains. 
The code $\phi_{t+1}$ is defined on the original (t+1)-trains as follows: We start by selecting one original ( $t+1)$-locomotive $A_{t+1}$ and one original ( $\left.t+1\right)$-caboose $B_{t+1}$. Then $\phi_{t}\left(A_{t+1}\right)$ and $\phi_{t}\left(B_{t+1}\right)$ are well defined $\left(A_{t+1}\right.$ and $B_{t+1}$ like all $(t+1)$-wagons are concatenations of some number of original $t$-trains). We transform all the $(t+1)$-trains by applying to each of them the code $\phi_{t}$ and then the following two modifications:

(A) We remove all symbols from the top and middle row of the locomotive and place them (preserving the order) in the central cells of the following $2 l_{t}$ wagons. Similarly, we remove all symbols from the top and middle row of the caboose and place them (preserving the order) in the central cells of the preceding $2 c_{t}$ wagons. By (1') and (6'), there are enough wagons in each $(t+1)$-train, moreover the central wagon will not be used, hence its central cell will remain empty.

(B) We replace the locomotive by $\phi_{t}\left(A_{t+1}\right)$ and the caboose by $\phi_{t}\left(B_{t+1}\right)$.

Note, that by ( $\left.7^{\prime}\right)$, the above code does not alter the bottom row. The so defined map $\phi_{t+1}$ is a 1-1 correspondence between original $(t+1)$-trains and the newly obtained $(t+1)$-trains, which we now call regular. During the modifications (A) and (B) each component (regular) $t$-train of the ( $t+1)$-train can be either left unaffected or replaced by another regular $t$-train (modification (B)), or it can happen that a letter will be inserted into its central cell (modification (A)). A $t$-train differing from a regular one in having the central cell occupied will be called an irregular t-train. In this notation, every regular $(t+1)$-train is a concatenation of regular and irregular $t$-trains. Every regular $(t+1)$-train has the "standard" locomotive $\phi_{t}\left(A_{t+1}\right)$ and the "standard" caboose $\phi_{t}\left(B_{t+1}\right)$.

As easily seen, $\phi_{t}$ converge pointwise to an invertible and action preserving map $\phi$ on $\tilde{X}^{\prime} \subset \tilde{X}$, defined as the set of such points that every coordinate falls into at most finitely locomotives and cabooses (i.e., for finitely many indices $t$ ). Namely, for such points every position can be affected by at most finitely many modifications (B); the modifications (A) never change a position more than once. The set $\tilde{X}^{\prime}$ is obviously shift invariant. It is also nonempty: note that there exists a point such that for every $t$ the zero coordinate falls in the central wagon of a $t$-train. (In fact, $\tilde{X}^{\prime}$ is residual, moreover, it has full measure for every invariant measure on $\tilde{X}$; this follows easily from the fact that $\left(p_{t}\right)$ grows to $\infty$.) For us it is essential that $\tilde{X}^{\prime}$ is dense in $\tilde{X}$. We let $Y=\overline{\phi\left(\tilde{X}^{\prime}\right)}$. By approximation, the bottom row of every $y \in Y$ is an element of $\tilde{Z}$, and for each $t \in \mathrm{N}, y$ is a concatenation of regular and irregular $t$-trains.

We are now in a position to define the factor map $\rho: Y \rightarrow Z$. For this we want to be able, for any $y \in Y$, to locate $\rho(y)$ in $Z$ up to $\epsilon_{t}$ accuracy knowing 
only the $t$-train of $y \in Y$ which covers the coordinate 0 . More precisely, we will assign a point $z_{c} \in Z$ to every $t$-train $c=c[0, k]$ of $Y$. We will also make sure that the above assignments converge, i.e., we will assure that if $d=d[0, l]$ is a $(t+1)$-train of $Y$ in which $c$ appears, say, $d[r, r+k]=c$, then for each $n$, $0 \leq n \leq k$ we have

$$
\operatorname{dist}\left(T^{n+r}\left(z_{d}\right), T^{n}\left(z_{c}\right)\right)<2 \epsilon_{t} .
$$

Once this is done, we define the map $\rho$ by the formula

$$
\rho(y)=\lim T^{n_{t}}\left(z_{c_{t}}\right),
$$

where, for each $t, y\left[-n_{t}, k_{t}-n_{t}\right]=c_{t}$ is a $t$-train (the $t$-train covering the coordinate 0 in $y$ ). Continuity of such a map follows immediately from summability of the sequence $\epsilon_{t}$. Later we will also check that $T \rho=\rho S$. We proceed with the assignment as follows:

Let $c$ be a (regular or irregular) $t$-train appearing in $Y$. If $c$ is regular then $\phi_{t}^{-1}(c)$ is well defined and it is an original $t$-train. If $c$ is an irregular $t$-train then we can easily produce a regular one from it by emptying the central cell in the middle row. By $\phi_{t}^{-1}(c)$ we shall mean the preimage by $\phi_{t}$ of the so obtained regular $t$-train. In either case, we select a point $\tilde{x}_{c} \in \tilde{X}$ in which $\phi_{t}^{-1}(c)$ appears at the coordinate 0 , and we let $z_{c}=\tilde{\pi}\left(\tilde{x}_{c}\right)$. It remains to check the convergence condition. Let $d$ be a $(t+1)$-train in which $c$ appears, $d[r, r+k]=c$. We consider separately two cases:

(a) $c$ falls neither into the locomotive nor caboose of $d$.

Then $\phi_{t}^{-1}(c)$ appears in $\phi_{t+1}^{-1}(d)$ at the same position $r$. Thus $z_{c}$ and $T^{r}\left(z_{d}\right)$ are images by $\tilde{\pi}$ of two points $\tilde{x}_{c}$ and $S^{r}\left(\tilde{x}_{d}\right)$ having the same $t$-train $\phi_{t}^{-1}(c)$ starting at zero. The application of (10) to $S^{n}\left(\tilde{x}_{c}\right)$ and $S^{n+r}\left(\tilde{x}_{d}\right)(0 \leq n \leq k)$ yields (11), hence ends this case.

(b) $c$ falls into either the locomotive or caboose of $d$.

We proceed for the case of a locomotive. Since $d$ has the "standard" locomotive $\phi_{t}\left(A_{t+1}\right), \phi_{t}^{-1}(c)$ is part of $A_{t+1}$ and it may not appear in the original locomotive of $\phi_{t+1}^{-1}(d)$. But $A_{t+1}$ is also an original locomotive, so there exists a point $\tilde{x}_{0} \in \tilde{X}$ which has this locomotive located at the position $-r$. Then $\phi_{t}^{-1}(c)$ appears in $\tilde{x}_{0}$ starting at zero, hence we can apply (10) to the pair $\tilde{x}_{c}$ and $\tilde{x}_{0}$. Denoting $z_{0}=\tilde{\pi}\left(\tilde{x}_{0}\right)$, we obtain

(12) $\operatorname{dist}\left(T^{n}\left(z_{c}\right), T^{n}\left(z_{0}\right)\right)<\epsilon_{t}$ for $0 \leq n \leq k$.

On the other hand, both $\tilde{x}_{0}$ and $S^{r}\left(\tilde{x}_{d}\right)$ have (possibly different, but never mind) $(t+1)$-locomotives at the position $-r$. Shifting each of them by $n$ 
and applying (8), we obtain $T^{n}\left(z_{0}\right) \in T^{n}\left(\overline{V_{t}}\right)$, and $T^{n+r}\left(z_{d}\right) \in T^{n}\left(\overline{V_{t}}\right)$. For $0 \leq n<p_{t}$, the application of (4) yields that

(13) $\operatorname{dist}\left(T^{n}\left(z_{0}\right), T^{n+r}\left(z_{d}\right)\right)<\epsilon_{t}$.

Combining (12) and (13) we obtain (11), and the convergence condition is verified.

It follows immediately from the definition of $\rho$ that the condition $T \rho=\rho S$ holds at such points of $Y$ where the zero coordinate is not the last one in a $t$-train for infinitely many indices $t$. But such points are dense in $Y$, hence, by continuity, $\rho$ preserves the action.

Finally, we need to show that the map $\rho$ provides an almost $1-1$ extension, by finding an element of $Z$ with a one-point preimage. It is easy to see that the point $z_{0}$ (the intersection of the sequence $\left(V_{t}\right)$ ) satisfies this condition; by (9), and because $z_{0}$ is in the interior of each $V_{t}$, each point in the preimage must have a $t$-train starting at zero in the bottom row. But there is only one such point in $Y$, namely the one which has the "standard" locomotives extending to the right, and the "standard" cabooses extending to the left from the zero coordinate.

REMARK 4.2. Using the above construction we can completely omit the assumptions made in [6] on the minimal flow $(Z, T)$. The proofs of all theorems there can be adapted accordingly. The full strength version is stated below. By a Borel $^{*}$ isomorphism between two flows $(X, T)$ and $(Y, T)$ we shall understand a Borel measurable invertible and action preserving map $\phi: X^{\prime} \rightarrow Y^{\prime}$ between sets $X^{\prime} \subset X$ and $Y^{\prime} \subset Y$, both of mass 1 for any invariant measure on the respective spaces, such that the associated map between the sets of invariant measures is an affine homeomorphism in the weak* topology.

THEOREM 4.3 (cf. [9] and [6]). Let $(X, T)$ be an arbitrary extension of a minimal non-periodic dynamical system $(Z, T)$ (we denote the corresponding factor map by $\pi_{X}$ ). Then $(X, T)$ is Borel ${ }^{*}$ isomorphic (via a map denoted by $\phi)$ to some minimal dynamical system $(Y, T)$ which is a topological almost 1-1 extension of $(Z, T)$ (we denote the corresponding factor map by $\left.\pi_{Y}\right)$, and the following diagram commutes:

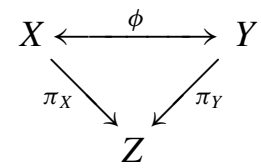

If $(X, T)$ is a subshift over an alphabet $\Sigma$ then $(Y, T)$ can be obtained also in a form of a subshift over the same alphabet $\Sigma$. 


\section{Examples of factors of Toeplitz flows}

Applying Theorems 3.2 and 4.1, and using the well known fact that a factor of an odometer is again an odometer, we give the following characterization of flows which are factors of Toeplitz flows:

THEOREM 5.1. A dynamical system $(X, T)$ is a factor of a Toeplitz flow if and only if it satisfies the following three conditions

(a) $(X, T)$ is minimal,

(b) $(X, T)$ is an almost $1-1$ extension of an odometer,

(c) $(X, T)$ has a symbolic extension.

We will give soon a practical condition equivalent to the conjunction of (a) and (b). But first we need to recall one of the standard methods of producing almost automorphic flows (cf. [12], [5] and [3]). Let $G$ be a compact monothetic group. We can view $Z$ as a subset of $G$ (by identifying the multiples of the topological generator with the integer coefficients). This induces on $\mathrm{Z}$ a new (precompact) topology. Let $K$ be some compact space and let $f: G \rightarrow K$ be a function whose restriction to $Z$ is continuous in this new topology. Finally, we let $\left(X_{f}, S\right)$ be the shift orbit closure of $(f(n))_{n \in Z}$ in $K^{Z}$. In order to have $\left(X_{f}, S\right)$ an extension of $(G, R)$ we need one more condition: Let $F$ denote the closure of the graph of $f \mid Z$ in $G \times K$. For each $g \in G$ let $F_{g}=\{k \in K:(g, k) \in F\}$. Note, that for every $n \in \mathrm{Z}$ we have $F_{n}=\{f(n)\}$. We say that $f$ is invariant under the rotation by $h(h \in G)$, if $F_{g+h}=F_{g}$ for every $g \in G$. If no such $h$ exists, then we say that $f$ is invariant under no rotations.

THeOREM 5.2. Let $(G, R)$ be a minimal rotation of a compact monothetic group, and let $(X, T)$ be a dynamical system. Then $(X, T)$ is a minimal almost $1-1$ extension of $(G, R)$ if and only if it is topologically isomorphic to a flow $\left(X_{f}, S\right)$ (as defined above), where $f$ is invariant under no rotations.

Proof. First consider the flow $\left(X_{f}, S\right)$. By definition, for every point $x \in$ $X_{f}$ there exists a sequence $n_{k}$ such that $x(n)=\lim _{k} f\left(n+n_{k}\right)$ for each $n \in Z$. Then $x(n) \in F_{n+h}$ for each $n$ if $h$ is a cluster point in $G$ of the sequence $\left(n_{k}\right)$. As is not hard to see, for every such $h$ the set of pairs $(n+h, x(n))(n \in Z)$ is dense in $F$. But $F$ is invariant under no rotation, hence $h$ is uniquely determined for every $x$. We omit the standard verification that the map $x \rightarrow h$ is continuous and preserves the action. It is also clear, that the unity $0 \in G$ is assigned to $f \mid Z$ and to no other element of $X_{f}$. Thus $\left(X_{f}, S\right)$ is an almost 1-1 extension of $(G, R)$. Minimality of $\left(X_{f}, S\right)$ is now immediate, because every invariant subset of $X_{f}$ must contain at least one point in the preimage of 0 , i.e., $f$, and hence its entire orbit closure, i.e., $X_{f}$. 
For the converse, suppose that $(X, T)$ is an almost 1-1 extension of $(G, R)$ via a map $\pi$. We can also assume (by rotating if necessary the group $G$ ) that 0 has a one-point preimage by $\pi$. Then the same holds for all elements of the orbit of 0, i.e., for the elements of Z. We simply let $K=X$ and for each $g \in G$ we choose $f(g)$ to be any element of $\pi^{-1}(g)$. It is easy to verify that the inverse to a continuous map between compact sets is continuous on the set of points having one-point preimages, hence $f$ is continuous on $Z$. The topological isomorphism between $\left(X_{f}, S\right)$ and $(X, T)$ is provided by the projection onto the coordinate 0 .

The condition (c) of Theorem 5.1 is by itself an interesting subject of investigation. Mike Boyle has an example of a finite entropy system which doesn't have this property ([1], see also [2]). Very likely, using a method of producing minimal flows based on the construction by S. Williams ([14]), one could obtain a minimal such example. On the other hand, it is known ([1], [2]) that any zero entropy system has a symbolic extension. ${ }^{1}$ In the following examples we avoid problems with the condition (c) by using only zero entropy flows.

If the function $f$ appearing in the definition of $\left(X_{f}, S\right)$ has the property that $F$ assumes one-point values on a full measure set in $G$ then $\pi$ is $1-1$ on a full measure set, hence $\left(X_{f}, S\right)$ is strictly ergodic and measure theoretically isomorphic to $(G, R)$, and thus has topological entropy zero.

Using the above methods we will now provide examples of minimal zero entropy almost 1-1 extensions of adding machines, i.e., by Theorem 5.1, flows which are factors of Toeplitz flows.

Let $G$ denote an odometer group.

Example 5.3. Consider $f: G \rightarrow I$, where $I=[-1,1]$, having a unique discontinuity point $g_{0} \in G \backslash Z$, and such that $F_{g_{0}}=I$ (i.e., behaving at $g_{0}$ like the function $\sin \frac{1}{x}$ at zero). The flow $\left(X_{f}, S\right)$ has all required properties. The fibers $\pi^{-1}(g)$ are either singletons or intervals. This provides an example of a non-totally disconnected factor of a Toeplitz flow.

The following example provides a complete answer to the questions concerning possible factors of Toeplitz flows raised in [10].

Example 5.4. We can construct a function as above, with $I$ replaced by the classical Cantor set $C$. This time we obtain of a totally disconnected factor of a Toeplitz flow.

Observe the points from the fiber $\pi^{-1}\left(g_{0}\right)$. These are sequences differing only at the position zero, where all values from $C$ can be assumed. Thus we can index them by these values: $\pi^{-1}\left(g_{0}\right)=\left\{x_{c}: c \in C\right\}$. It is now seen that the

\footnotetext{
${ }^{1}$ The flows admitting a symbolic extension have been recently characterized in [4].
} 
flow is not expansive, because the points $S^{n}\left(x_{c}\right), S^{n}\left(x_{c}^{\prime}\right)$ are close to each other at all times $n \in \mathrm{Z}$ for close values of $c$ and $c^{\prime}$. Thus $\left(X_{f}, S\right)$ is not symbolic, in particular it is not a Toeplitz flow. Clearly, this flow is not an odometer, because no odometer is an almost $1-1$ extension of another.

We claim that our flow is not even a minimal product of a Toeplitz flow with another odometer. We have to say a few words about such products. If $(X, S)$ is a Toeplitz flow over an odometer $\left(G_{1}, R_{1}\right)$, and $\left(G_{2}, R_{2}\right)$ is another odometer, then the product $(Y, T)=\left(X \times G_{2}, S \times R_{2}\right)$ is minimal if and only if the groups $G_{1}$ and $G_{2}$ are orthogonal, i.e., if their dual groups, viewed as discrete subgroups of the torus, have trivial intersection $\{1\}$. Such a product is an almost 1-1 extension over the odometer $(G, R)=\left(G_{1} \times G_{2}, R_{1} \times R_{2}\right)$. It is no longer expansive; the consecutive images of two points of the form $\left(x, g_{1}\right)$ and $\left(x, g_{2}\right)$ will remain at the same distance as $g_{1}$ and $g_{2}$. Nevertheless, let $y=\left(x_{1}, g_{1}\right)$ and $y^{\prime}=\left(x_{2}, g_{2}\right)$ be two different points of the product belonging to the same fiber over $(G, R)$. Then obviously $g_{1}=g_{2}$, hence $x_{1} \neq x_{2}$, which implies that the consecutive images of $y$ and $y^{\prime}$ cannot stay close at all times, i.e., behave as $x_{c}$ and $x_{c}^{\prime}$ constructed above. This proves our claim.

Finally, we can also easily produce flows with a large variety of fibers.

EXAMPLE 5.5. Let $\left(K_{n}\right)_{n \in \mathrm{N}}$ be any countable family of compact metric spaces represented as subsets of the Hilbert cube $K$. Let $f_{n}$ be a function on $G$ into $K$ which has only one discontinuity at a point $g_{n} \in G \backslash Z$, and such that $F_{g_{n}}=K_{n}$ (here $F$ denotes the appropriate set for $f_{n}$ ). We also assume that the points $g_{n}$ have pairwise disjoint orbits. Then we define $f: G \rightarrow K$ as $f=$ $\Sigma_{n} \frac{f_{n}}{2^{n}}$ (addition is defined coordinatewise in the Hilbert cube). This function has discontinuities only at the points $g_{n}$, hence it satisfies all requirements of the construction of $\left(X_{f}, S\right)$. It is seen that $\pi^{-1}\left(g_{n}\right)$ is homeomorphic to $K_{n}$, i.e., that $\left(X_{f}, S\right)$ has the designed fibers.

\section{REFERENCES}

1. Boyle, M., The residual entropy of a dynamical system, presented in Sapporo, 1992.

2. Boyle, M., Fiebig, D. and Fiebig, U., Residual entropy, conditional entropy and subshift covers, Forum Math., to appear.

3. Downarowicz, T., How a function on a zero-dimensional group $\Delta_{a}$ defines a Toeplitz flow, Bull. Pol. Acad. Sci. 38 (1990), 219-222.

4. Downarowicz, T., Entropy of a symbolic extension of a totally disconnected dynamical system, Ergodic Theory Dynam. Systems 21 (2001), 1051-1070.

5. Downarowicz, T. and Iwanik, A., Quasi-uniform convergence in compact dynamical systems, Studia Math. 89 (1988), 11-25.

6. Downarowicz, T. and Lacroix, Y., Almost 1-1 extensions of Furstenberg-Weiss type, Studia Math. 130 (1998), 149-170

7. Eberlein, E., Toeplitz folgen und Gruppentranslationen, Thesis, Erlangen, 1970. 
8. Furstenberg, H., Recurrence in ergodic theory and combinatorial number theory, Princeton Univ. Press, Princeton, N.J., 1981.

9. Furstenberg, H. and Weiss, B., On almost 1-1 extensions, Israel J. Math. 65 (1989), 311-322.

10. Gjerde, R. and Johansen, O., Bratteli-Vershik models for Cantor minimal systems: applications to Toeplitz flows, Ergodic Theory Dynam. Systems 20 (2000), 1687-1710.

11. Jacobs, K. and Keane, M., 0-1 sequences of Toeplitz type, Z. Wahr. 13 (1969), 123-131.

12. Markley, N. G., Substitution-like minimal sets, Israel J. Math. 22 (1975), 332-353.

13. Markley, N. G. and Paul, M. E., Almost automorphic symbolic minimal sets without unique ergodicity, Israel J. Math. 34 (1979), 259-272.

14. Williams, S., Toeplitz minimal flows which are not uniquely ergodic, Z. Wahrsch. Verw. Gebiete 67 (1984), 95-107.

INSTITUTE OF MATHEMATICS

TECHNICAL UNIVERSITY

WYBRZEŻE WYSPIAŃSKIEGO 27

50-370 WROCŁAW

POLAND

E-mail: downar@im.pwr.wroc.pl
UNIVERSITÉ DE PICARDIE JULES VERNE FACULTÉ DE MATHÉMATIQUES ET INFORMATIQUE 33, RUE SAINT LEU 80039 AMIENS CEDEX 01 FRANCE

E-mail: fd-math@u-picardie.fr 